\title{
REVIEWER LIST 1996
}

We extend our sincerest gratitude to the following reviewers who have contributed their time and expertise to our journal. This list includes all individuals who provided or delegated reviews for papers appearing in Volume 4, Issues 1-4, of the Journal of Intelligent \& Fuzzy Systems.

Mo Jamshidi and Timothy J. Ross, Editors-in-Chief Karen Koletar, Editorial Manager

Nahrul Alang-Rashid

Institut Penyelidikan Teknologi Nuklear (MINT)

Mohammad Akbarzadeh

University of New Mexico

N.O. Attoh-Okine

Florida International University

B. M. Ayyub

University of Maryland

Ali Bahrami

Rhode Island College

Marik Balazinski

Ecole Polytechnique de Montreal

R. Bertin

Laboratoire d'Automatique et Productique

de l'Universite de Bordeaux

Dr. James C. Bezdek

University of West Florida

Zeungnam Bien

Korea Advanced Institute of Science \& Technology (KAIST)

David I. Blockley

University of Bristol

Slavka Bodjanova

Texas A\&I University

Pierre Borne

Ecole Centrale de Lille

James J. Buckley

University of Alabama at Birmingham

James M Carson

University of New Mexico

Chi-Wei Chang

Texas A\&M University

Myung-Geun Chun

Samsung Electronics

R. Colbaugh

New Mexico State University

P.K. Dash

National University of Singapore

Ahmed M. Darwish

Cairo University

Joe DeWitte

Naval Surface Warfare Center

Mark E. Dretar

Bell Helicopter Textron, Inc.

Dimetar P. Filev

Ford Motor Company

Fu Guoyao

Nanjing Gas Turbine Research Institute

Liu Geng

University of Bristol

Vipul Gupta

University of New Mexico

Hisao Ishibuchi

University of Osaka Prefecture

Mo Jamshidi

University of New Mexico

John Dewey Jones

Simon Fraser University

Nicolaos Karayiannis

University of Houston

Waldemar Karwowski

University of Louisville

Nasser Kehtarnavaz

Texas A\&M University

James Keller

University of Missouri-Columbia

L. Ken Keys,

Cleveland State University

Jong-Hwan Kim

Korea Advanced Institute of Science and Technology (KAIST)

Seong-Gon Kong

Soongsil University

Minho Lee

Korea Advanced Institute of Science and Technology (KAIST)

Paul J.A. Lever

The University of Arizona

Soo-Young Lee

Korea Advanced Institute of Science and Technology (KAIST)

Xiaoqing Liu

Texas A\&M University

Gui Zhang Lu

Nankai University

Caro Lucas

University of Tehran 
George F. Luger

University of New Mexico

Rinaldo C. Michelini

University of Genoa

Hidetoshi Miike

Yamaguchi University

Tsutomu Miki

Kyushu Institute of Technology

Kyung Chan Min

Yonsei University

John Mo

Royal Melbourne Inst. of Technology

Bhaba Krishna Mohanty

Indian Institute of Management

Kevin L. Moore

Idaho State University

Oscar Moreira-Tamayo

Texas A\&M University

Gary P. Moynihan

The University of Alabama

D. Subbaram Naidu

Idaho State University

Nikhil R. Pal

Indian Statistical Institute

Cheol Hoon Park

Korea Advanced Institute of Science and Technology (KAIST)

Dong-Jo Park

Korea Advanced Institute of Science and Technology (KAIST)

Witold Pedrycz

University of Manitoba

P. K. Rajan

Tennessee Technological University

Daniel W. Repperger

Armstrong Laboratory, Wright-Patterson AFB

Timothy Ross

University of New Mexico

Thomas Runkler

SIEMANS

Harold Szu

Naval Surface Warfare Center

Hiroyuki Tamura

Osaka University
Sie-Keng Tan

National University of Singapore

Christoph Thomas

SIEMANS

Andre Titli

Laboratoire d'Analyse

et d'Architecture des Systemes

Amy J. C. Trappey

National Tsing Hua University

Poyu Tsou

Ford Motor Company

Eddie Tunstel

University of New Mexico

Ismail Burhan Turksen

University of Toronto

Ching-Yu Tyan

Duke University

Nader Vadiee

University of New Mexico

Andy Vann

University of Bristol

Erol Varoglu

Forintek Canada Corp.

Claude Vibet

Massy, France

Fei-Yue Wang

The University of Arizona

Paul P. Wang

Duke University

Hong Quan Yang

Simon Fraser University

James T. P. Yao

Texas A\&M University

Hao Ying

University of Texas / Medical Branch

Masataka Yoshimura

Kyoto University

Renhong Zhao

University of Cincinnati

Donald S. Zinger

Northern Illinois University

Alessandro Zorat

University of Trento 